\title{
Metrological evaluation of laser scanner integrated with measuring arm using optical feature-based gauge
}

\author{
Cuesta, E.; Alvarez B.J.; Martinez-Pellitero, S.; Barreiro, J., Patiño, H.
}

\begin{abstract}
This work deals with the searching of metrological limits with which the laser scanners mounted on coordinate measuring arms (AACMMs or CMAs) are able to evaluate dimensional and geometric tolerances (GD\&T). For this purpose, a novel feature-based gauge for optical sensors is used. This gauge incorporates different types of geometrical entities, perfectly adapted for several GD\&T evaluations. In addition, these entities are optically functional so that they are captured from the laser sensors mounted in AACMM. The gauge is equipped with a set of "canonical" entities of ceramic type, manufactured with high dimensional accuracy to materialize a multitude of GD\&T tolerances. Regarding the metrological evaluation, the measurements obtained with the laser scanner are compared with the previously calibrated measurements of the gauge, calibration that has been performed using contact probing in a CMM. Although in this research a rather obsolete model of laser scanner is used, the methodology is totally valid for any sensor mounted in AACMM, obtaining a high range of traceable values.
\end{abstract}

One issue that has received special attention is the control of variability produced by a manual scanning operation. To this end, research is approached from a twofold perspective: on one hand, an initial study to determine the best scanning strategy allowing a good coverage of each entity surface, and, on the other hand, a statistical analysis from a high number of repetitions of a complete measurement routine of the feature-based gauge. Thus, reliable dispersion values can be offered not only for different types of GD\&T evaluations (form, dimensions, etc.) but also for the same GD\&T evaluation over the same type of entity.

Although the work proposes precision values depending on the type of GD\&T being analyzed, it also proposes a novel reliable method of calculation of the probing error for a laser scanner mounted on an AACMM.

Keywords

3D Optical scanner; Coordinate Measuring Arm; Metrological evaluation; Feature-based gauge; Laser Triangulation Sensor

Taxonomy

Laser Scanning, Optical Imaging Techniques 


\section{Introduction}

The use of laser triangulation sensors has initially been reserved for typical reverse engineering tasks. However, an increasing trend pursues to extend its field of application to the world of dimensional metrology. Unfortunately, the equipment and the software are not specifically designed to measure dimensional and geometric tolerances (GD\&T), even though the equipment where they are mounted (AACMMs) are oriented for these GD\&T inspection tasks, especially when using touch probing.

This work presents an experimentation aimed at analysing the measurement accuracy of Laser Triangulation Sensors mounted on Portable Coordinate Measuring Arms (CMAs). This measuring device is generally used to capture high-density point clouds over the surfaces of an object (in the present case the object will be a featurebased artefact) during short periods of time [1, 2]. Nevertheless, the evaluation or quantification of the measurement accuracy is highly difficult due to a series of errors and factors involved. In fact, several researchers have studied this issue in recent years, for Laser sensors either mounted on Coordinate Measuring Machines (CMMs) where the trajectories and orientations of the sensor are automated [3-8] or mounted on Portable Coordinate Measuring Arms [9-12] where the sensor is handled manually. Some researchers have identified and studied factors of influence such as: the geometric parameters of the sensor, the qualification of the sensor [8], the relative orientation between the object, the laser beam and the CCD [11,13], the scanning strategy and point cloud density [11], or even factors related with the surface finish of the part (colour, roughness, brightness, reflectivity, etc.) $[7,10,14,15]$. All these factors are relevant for the quantification of the sensor accuracy, which is accomplished by means of the metrological evaluation of the dimensional and geometrical tolerances constraining a reference artefact, provided with spherical and planar features. The evaluation involves comparing the measurements obtained with the laser sensor and the reference values of those tolerances, previously obtained by means of a high accuracy contact sensor mounted on a CMM [12]. A common practice in the metrological assessment of the accuracy of this type of equipment is based on the application of standards, namely, IS0 10360-8:2013 and VDI/VDE 2617. In the application of these standards [15, 16] the objective is to determine the probing and the volumetric error similarly to what is accomplished for the evaluation of contact sensors, but now with optical sensors mounted on machines of Cartesian structure.

All of the previous studies reveal the absence of standardized procedures for the assessment of conformity of laser sensors, in order to check the quality of point clouds or to assign uncertainties to the measurements performed with this technology. In this research, a laser triangulation sensor model G-Scan, mounted on a portable coordinate arm model Romer Sigma (Hexagon Metrology), will be used.

Undoubtedly, in the last years, the equipment directly related with non-contact reverse engineering have grown substantially. The possibility of capturing high quality dense point clouds over different surfaces in short periods has increased the versatility of these instruments. Furthermore, the capability of modern software tools of transforming point clouds into surfaces have helped to their industrial deployment. Amongst others, the following applications stand out: surface reconstruction, CAD comparison, statistical analysis, inspection and metrological verification of prototypes. However, the optical nature of this type of equipment involves a series of disadvantages such as the aforementioned ones (sensor parameters, light reflections, sensor resolution, part colour and "edge effect" on the boundaries of the surfaces). In any case, the study must also consider the characteristics of the different sensor models, as well as the diverse reverse engineering software applications for the treatment of point clouds and the way used for reconstructing the surfaces [17]. Even when the method for capturing point clouds is always based on triangulation, these systems can present different angles of triangulation, capture densities, focal distances, CCD resolutions, etc., which led to the consideration of different 3D laser scanners.

In this work, it is intended to fill the gap caused by the lack of universally accepted standards and procedures for the determination of the uncertainty of measurements derived from the point clouds.

Although this idea may be applied to other reverse engineering equipment, the experimentation included in this work is orientated to a specific laser sensor (chapter 2.1) evaluated with the aid of an artefact specifically designed for it (chapter 2.2) and a software for reverse engineering, i.e., 3DReshaper (Technodigit ${ }^{\circledR}$ ).

\section{Materials and methodology}

The evaluation is based on the verification of the laser sensor by measuring a reference gauge. This gauge presents suitable optical properties, and it is provided with a set of geometric features constrained by a wide series of dimensional and geometric tolerances: distances between features, feature sizes, form deviations or orientation 
tolerances (associated to a datum feature). This gauge is denominated feature-based gauge, and its concept was researched in precedent projects [18-20] leading to a European patent [21].

\subsection{Laser Triangulation Sensor (LTS)}

The equipment used during this research project consists of a laser triangulation sensor (R-Scan) and a portable coordinate arm, a six DOF model of Romer, with a measuring range of 1,8 $\mathrm{m}$ (spherical volume radius) and a weight of $4,6 \mathrm{~kg}$. In contact measurements the specifications given by the manufacturer for this CMA are: repeatability of $\pm 0,010 \mathrm{~mm}$ (sphere test) and of $\pm 0,018 \mathrm{~mm}$ (cone test, similar to the Single Point Articulation Test, SPAT defined in ISO 10360-12, Annex D). After calibration following internal evaluations similar to those included in the standard ISO 10360-12 [20], the length uncertainty was $\pm 0,042 \mathrm{~mm}$.

The R-Scan sensor mounted on the CMA may be configured through several parameters in order to optimize the measurement according to the geometry and material of the part to scan. Table 1 shows the main characteristics of the R-Scan Laser scanner.

Table 1. Laser Scanner available.

\begin{tabular}{ll}
\hline \multicolumn{2}{c}{ Laser Scanner (R-Scan) Technical specifications } \\
\hline Weight / dimensions & $600 \mathrm{~g} / 156 \times 72 \times 50 \mathrm{~mm}$ \\
Distance camera-measured surface & $124<\mathrm{d}<222 \mathrm{~mm}$ \\
Laser line maximum length & $110 \mathrm{~mm}$ \\
Plane laser angle (laser line generator) & $45^{\circ}$ \\
Minimum distance between 2 points without interpolation & $0.10 \mathrm{~mm}$ \\
Accuracy camera lens only (2 Sigma) & $0.07 \mathrm{~mm}$ \\
Maximum acquisition speed & 20 laser-lines/second \\
Plane laser wavelength & $635 \mathrm{~nm}$ \\
Maximum measured points per line & 640 \\
Maximum plane laser emission power & $5 \mathrm{Mw}$ \\
Laser type & Diode \\
Security (plane laser) IEC & Class 3a \\
\hline
\end{tabular}

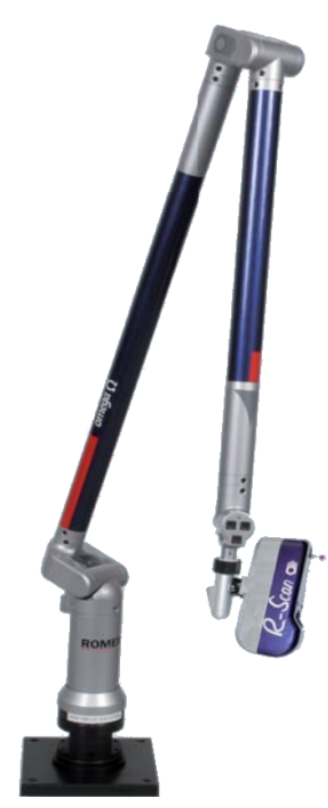

\subsection{Feature-based gauge for optical measurements}

The reference artefact chosen for studying the accuracy of LTS measurements is an experimental feature-based gauge derived from a previous research oriented to assess the accuracy of measurements made with contact sensors mounted on CMAs. The gauge satisfies to a great extent the specifications of the aforementioned patent $[20,21]$ regarding the aspects of design and utility considering its purpose for calibration and verification of CMAs. Now, the new version of the gauge is specifically developed for the assessment of optical systems for reverse engineering. In fact, its conception and development are oriented to the evaluation of LTS mounted on CMAs and structured light 3D scanners (photogrammetry by fringe projection pattern) [22].

The novel gauge presents as innovation a new support for the features made in carbon fibre of high Young modulus, which consists of two solid bars $(E=150 \mathrm{GPa})$ and a plate $(E=450 \mathrm{GPa})$ placed between the bars. In the upper part of this supporting plate there are located different geometric features like planar surfaces, external cylinders, internal cylinders, cones or spheres, all of them manufactured at high precision in matt white ceramic.

The gauge (Fig. 1) contains six prismatic inserts (whose nominal size is $50 \times 25 \times 25 \mathrm{~mm}^{3}$ ) made of a machinable ceramic called MACOR® [23], as well as for cylindrical inserts of the same material. The outer surfaces of the prismatic volumes constitute the planar entities. In addition, the four cylindrical inserts provide four cylindrical surfaces whose external nominal diameter is $40 \mathrm{~mm}$ and whose nominal height is $40 \mathrm{~mm}$. Each one of the two cylindrical inserts more distant apart contain two internal bored cylindrical holes whereas in the other two inserts there are two internal conical surfaces. The nominal angle (between two generatrix lines) of these internal cones is $48^{\circ}$, and the nominal diameter of their basis is $32 \mathrm{~mm}$. 


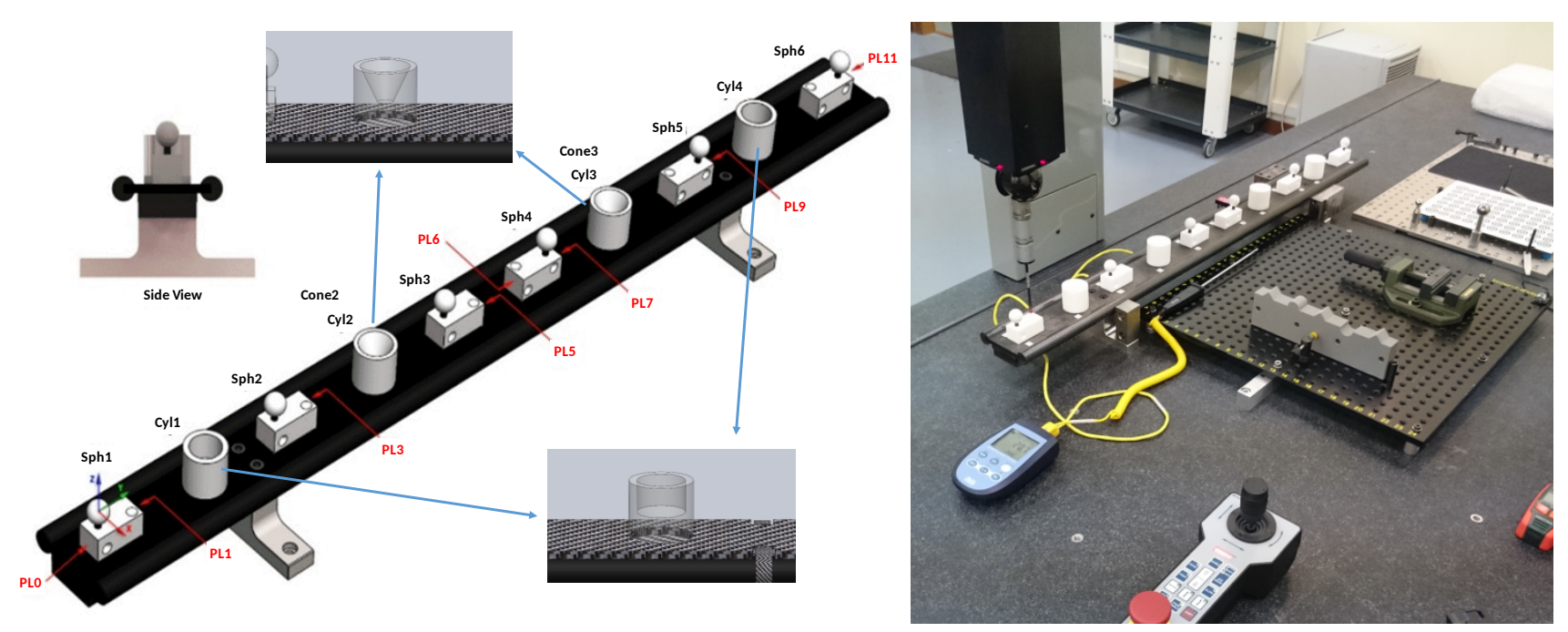

Fig. 1. Left.- CAD model of Optical feature-based gauge. Right.- Gauge calibrated at the CMM (DEA Global Image).

In the upper part of each prismatic volume (6 units) there is a precision sphere whose nominal diameter is $20 \mathrm{~mm}$, manufactured in a ceramic mixture of aluminium oxide $\left(\mathrm{Al}_{2} \mathrm{O}_{3}\right)$ and zirconium dioxide $\left(\mathrm{ZrO}_{2}\right)$. The spheres are located higher than the prismatic blocks in order to be probed by means of some cylindrical stems manufactured in carbon fibre.

The location of all these features along the length of the gauge may be observed in Fig. 1. The fixture that supports the feature-based gauge has been designed for clamping the gauge at the Bessel points (very close to the Airy points), aiming at minimizing the variation of the neutral fibre of the bi-supported beam. In fact, for certain measurements, the point selected for probing has been located as close to the neutral axis as possible.

Table 2. Gauge features and GD\&T dimensions materialized by those features.

\begin{tabular}{lll}
\hline Type of dimension & Feature (Evaluation) & Description \\
\hline Dimensional & Spheres & 6 Diameters of spheres $(\varnothing 20 \mathrm{~mm}), \mathrm{Sph} 1$ to Sph6 \\
& Outer cylinders & 4 Diameters of outer cylinders $(\varnothing 40 \mathrm{~mm})$, Cyl1 to Cyl4 \\
& Inner cylinders & 2 Diameters of inner cylinders $(\varnothing 30 \mathrm{~mm})$, Cyl1 and Cyl4 \\
& Inner cones & 2 Angles of inner cones $\left(48^{\circ}\right)$, Cone2 and Cone3 \\
\hline Distances & Spheres & 5 Distances between spheres centres: from Sph1 to i-th Spheres [200 950 mm] \\
& Planes & 7 Distances: from PL0 to PL1, PL3, PL5, PL7, PL9, PL11; and PL5-PL6 [50 990 mm] \\
& Outer cylinders & 3 Distances between cylinders axes: from Cyl1 to Cyl2, Cyl3, Cyl4 [200 700 mm] \\
& Inner cylinders & 1 Distance from the axis of Inner Cylinder 1 to the axis of Inner Cylinder 4 [700 mm] \\
\hline Form errors & Spheres & Sphericity (6 Spheres) \\
& Planes & Flatness (7 Planes) \\
& Outer cylinders & Cylindricity (4 Outer cylinders) \\
& Inner cylinders & Cylindricity (2 Inner cylinders) \\
& Inner Cones & Cone form error (Cone2 and Cone3) \\
\hline $\begin{array}{l}\text { Position or } \\
\text { orientation errors } \\
\text { (combined) }\end{array}$ & Perpendicularity & Adjacent planes (vertical plane 5 and horizontal planes 4-5) \\
& Parallelism & 7 Parallelisms (from Plane 0 to i-th planes) \\
\hline
\end{tabular}

The feature-based gauge has been calibrated by measurements performed on a Coordinate Measuring Machine (DEA Global Image, Hexagon Metrology), with a Maximum Permissible Error in length measurements (ISO 103602) of $\mathrm{MPE}_{E}[\mu \mathrm{m}]=2.2+0.003 \cdot \mathrm{L}, \mathrm{L}$ in $[\mathrm{mm}]$, that supposes an enough accuracy for the purpose of this research. Furthermore, a wide range of measuring techniques has been applied to compensate the usual errors committed during calibrations performed with CMMs, such as, for example: reversal measurements, multiposition measurements, high number of iteration in measurements (at least 12). Among others, the calibrated GD\&T dimensions were: diameter and cylindricity for outer and inner cylinders, diameter and sphericity of spheres, flatness of planes, distance between cylinder axes, distance between sphere centres, distance between parallel planes, angles and form error of cones, and others (Table 2). 


\subsection{Methodology for the metrological evaluation}

The methodology applied for the metrological evaluation of the feature-based gauge is summarised in Fig. 2. Once the gauge was manufactured and all of its parts were thoroughly assembled, the next step involved its calibration by measuring it with a CMM (Fig. 1 right). As a result of the calibration, a reference value was obtained for each one of the GD\&T dimensions considered in this study. Subsequently, the feature-based gauge was measured with the laser sensor mounted on the CMA to obtain the corresponding results of each of the same GD\&T dimensions (Fig. 3). Finally, for each GD\&T dimension, a comparison between the measured result obtained with the laser sensor and the reference value derived from the calibration with the CMM was performed. Such comparison served for verifying and validating the measurements performed with the laser sensor, as the reference values obtained with the CMM are characterized by a sensibly lower uncertainty.

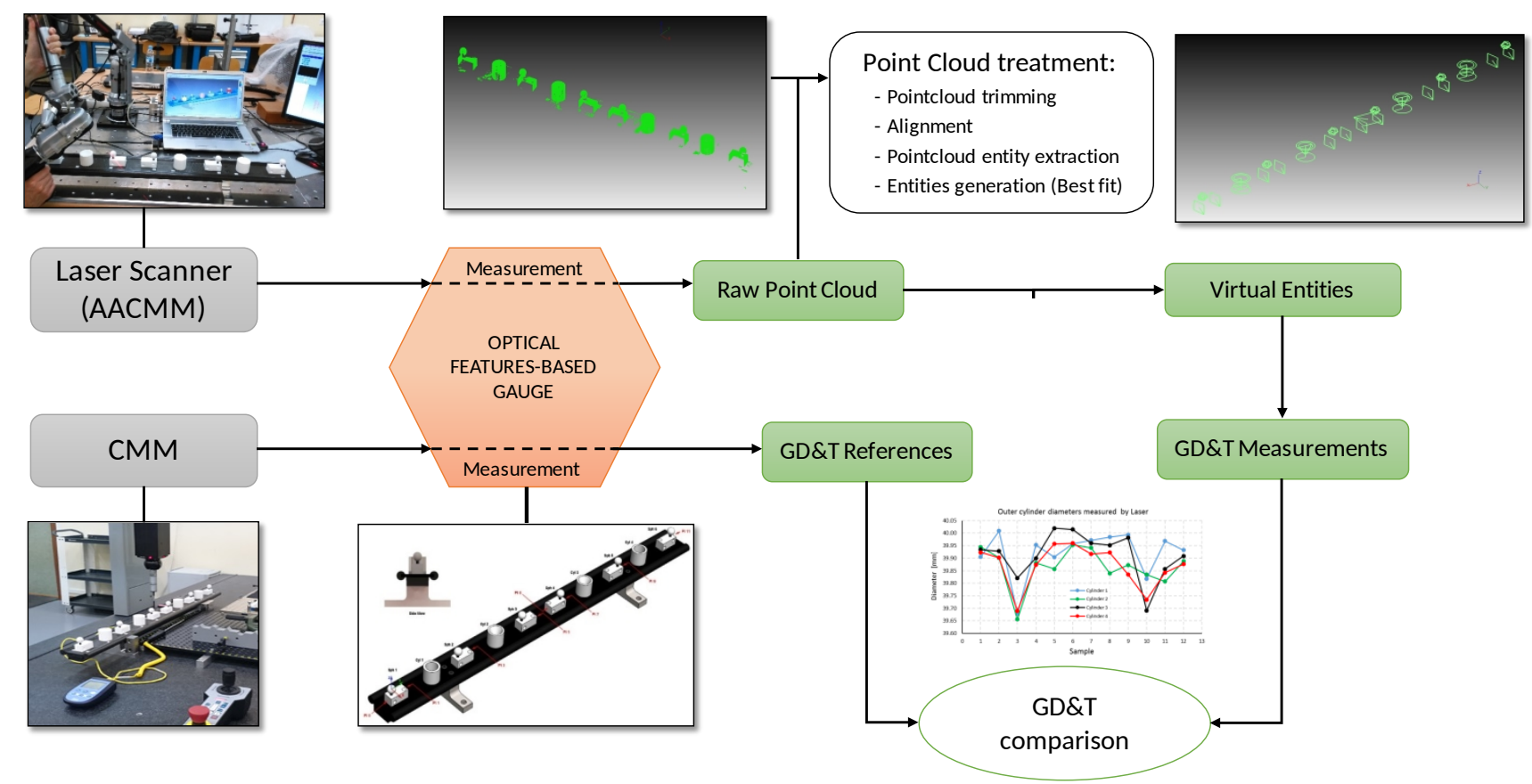

Fig. 2. Experimentation methodology.

The laser sensor technology and the geometry of the gauge have a direct influence on the measurement strategy to define for capturing the measured features. The objective is to obtain a widely covered surface during the manual acquisition of points and, at the same time, to avoid capturing surfaces of other features of no interest for the evaluation. This requirement has led to a previous study aiming at providing certain repeatability of the capturing process, maximizing the covered area over the surface and avoiding excessively large point clouds and lack of accuracy. The number of points is directly proportional to the number of "passes" of the laser beam over the surface. This previous study has allowed the definition of an optimal scanning strategy for each type of feature.

Apart from measuring each feature following a similar procedure (approximately the same number of passes) in order to minimize the influence of the operator in a manual process as the one studied in this paper, the obtained measurement results must be analysed statistically. To do this, 12 scanning measurements (tests) were carried out for all the gauge reference features.

For each of the 12 tests, a series of post-processing steps has been performed (see Section 3), from the initial cleaning of the acquired raw point clouds (filtering and aligning or registering the point clouds) until the building of virtual features that best match the point clouds, and the metrological study of these virtual features. In the last step, the measurement results obtained through the reconstructed software (3DReshaper) are compared with the reference values previously measured with the CMM. 


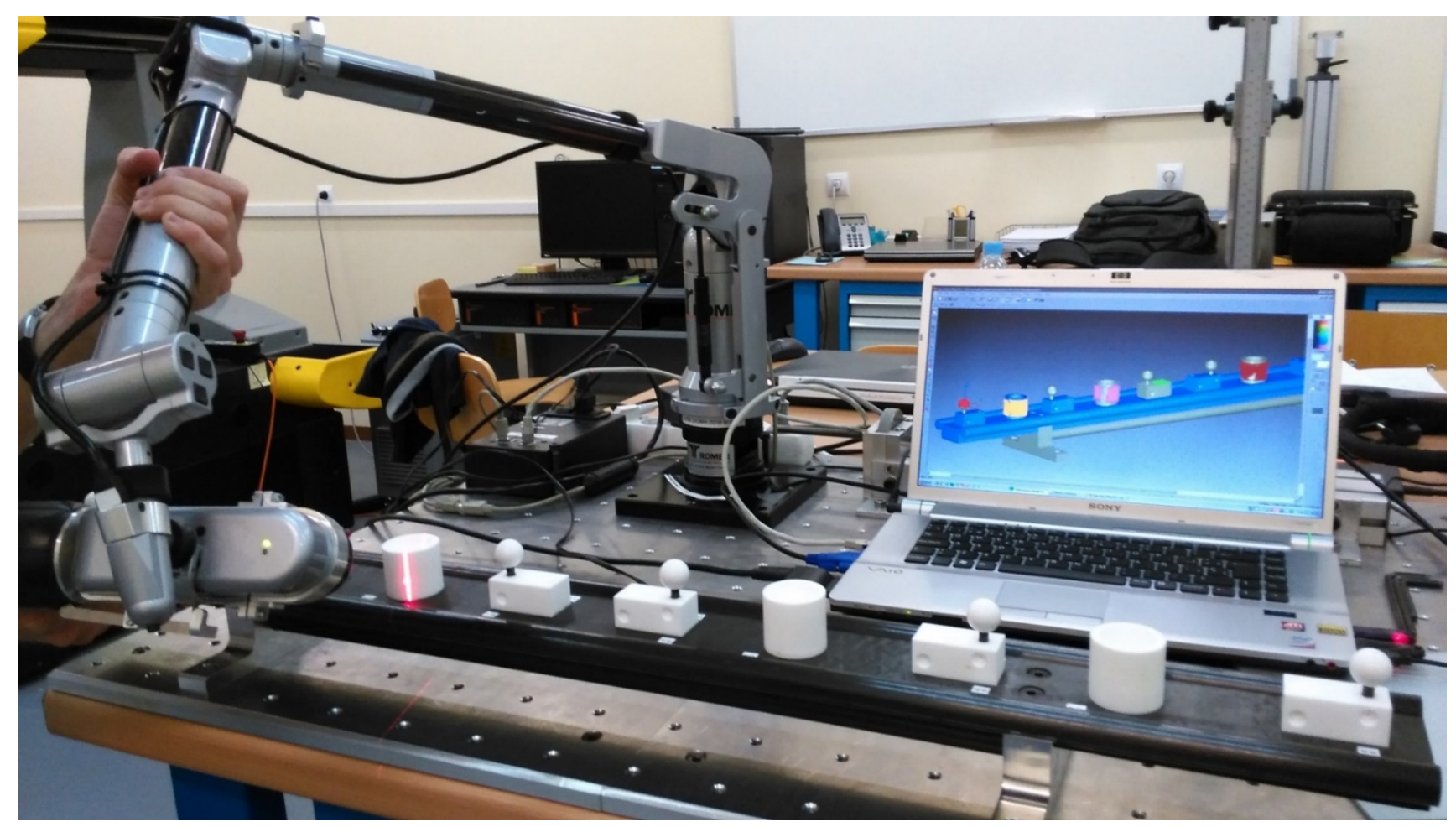

Fig. 3. Equipment \& gauge layout during two of the measuring tests.

Our approach is similar to the evaluation proposed by ISO, in the sense of separating the specific evaluation of the sensor, emulating the method of probing 25 points over a precision sphere, from the volumetric or global evaluation over reference features (cylinders, cones,...)

\section{Experimentation}

\subsection{Measurement of the gauge with the CMM. Reference measurement values}

Firstly, the feature-based gauge was calibrated with the aid of a Coordinate Measuring Machine (Fig. 1 right), leading to a set of measurement results (Table 3) that will be used as reference values for the evaluation of measurements performed with the laser sensor mounted on the CMA. During the calibration, several methods were applied to reduce the measurement errors arising in the use of CMMs thus obtaining reference values as close as possible to the actual values of the physical gauge. In this sense, a series of 12 measurements (tests) were carried out, varying the position of the gauge in the working volume of the CMM, and employing reversal techniques in the measurement related to each position.

Table 3. Reference measurements (CMM) of the Features-Based Gauge.

\begin{tabular}{|c|c|c|c|c|c|c|c|c|}
\hline & \multicolumn{2}{|c|}{ Features involved } & \multirow{2}{*}{$\begin{array}{l}\text { Nominal } \\
200,0000 \\
\end{array}$} & \multirow{2}{*}{$\begin{array}{c}\begin{array}{c}\text { CMM } \\
\text { result }\end{array} \\
200,0977\end{array}$} & & \multicolumn{2}{|c|}{ Features involved } & \multirow{2}{*}{$\begin{array}{c}\text { CMM resul } \\
0,0031 \\
\end{array}$} \\
\hline \multirow{13}{*}{ 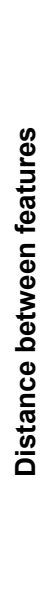 } & \multirow{5}{*}{ Spheres } & Sph1-Sph2 & & & \multirow{13}{*}{ 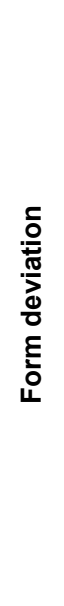 } & \multirow{6}{*}{ Spheres } & Sph1 & \\
\hline & & Sph1-Sph3 & 405,0000 & 405,1156 & & & Sph2 & 0,0013 \\
\hline & & Sph1-Sph4 & 535,0000 & 535,1301 & & & Sph3 & 0,0013 \\
\hline & & Sph1-Sph5 & 740,0000 & 740,0398 & & & Sph4 & 0,0018 \\
\hline & & Sph1-Sph6 & 940,0000 & 939,8675 & & & Sph5 & 0,0009 \\
\hline & \multirow{7}{*}{ Planes } & PL5-PL6 (int) & 50,0000 & 49,9256 & & & Sph6 & 0,0022 \\
\hline & & PL0-PL1 & 50,0000 & 50,0997 & & \multirow{4}{*}{ Outer cylinders } & Cyl1 & 0,0055 \\
\hline & & PL0-PL3 & 250,0000 & 250,1505 & & & Cyl2 & 0,0054 \\
\hline & & PL0-PL5 & 455,0000 & 455,2393 & & & Cyl3 & 0,0065 \\
\hline & & PL0-PL7 & 555,0000 & 555,2794 & & & Cyl4 & 0,0073 \\
\hline & & PL0-PL9 & 760,0000 & 760,2511 & & \multirow{2}{*}{ Inner cylinders } & Cyl1 & 0,0074 \\
\hline & & PL0-PL11 & 960,0000 & 960,1618 & & & Cyl4 & 0,0128 \\
\hline & Outer & Cyl1-Cyl2 & 200,0000 & 200,0644 & & Inner cones & Cone2 & $0,0243^{\circ}$ \\
\hline
\end{tabular}




\begin{tabular}{|c|c|c|c|c|c|c|c|c|}
\hline & \multirow[t]{2}{*}{ cylinders } & Cyl1-Cyl3 & 500,0000 & 500,0690 & & & Cone3 & $0,0221^{\circ}$ \\
\hline & & Cyl1-Cyl4 & 700,0000 & 700,1147 & & \multirow{8}{*}{ Planes } & PLO & 0,0034 \\
\hline & Inner cyl. & Cyl1-Cyl4 & 700,0000 & 700,1267 & & & PL1 & 0,0072 \\
\hline \multirow{14}{*}{ 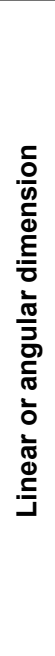 } & \multirow{6}{*}{ Spheres } & Sph1 & 20,0000 & 19,9999 & & & PL3 & 0,0054 \\
\hline & & Sph2 & 20,0000 & 20,0003 & & & PL5 & 0,0031 \\
\hline & & Sph3 & 20,0000 & 20,0018 & & & PL6 & 0,0077 \\
\hline & & Sph4 & 20,0000 & 19,9998 & & & PL7 & 0,0025 \\
\hline & & Sph5 & 20,0000 & 20,0020 & & & PL9 & 0,0065 \\
\hline & & Sph6 & 20,0000 & 20,0002 & & & PL11 & 0,0076 \\
\hline & & Cyl1 & 40,0000 & 40,0252 & $\approx$ & & PL5-PL6 & 0,0339 \\
\hline & Outer & Cyl2 & 40,0000 & 39,9809 & 茜 & & PL0-PL1 & 0,0175 \\
\hline & cylinders & Cyl3 & 40,0000 & 40,0266 & ฮे & & PL0-PL3 & 0,0408 \\
\hline & & Cyl4 & 40,0000 & 40,0053 & б & Parallelism & PL0-PL5 & 0,0398 \\
\hline & Inner & Cyl1 & 30,0000 & 29,4822 & సٓ & & PL0-PL7 & 0,0211 \\
\hline & cylinders & Cyl4 & 30,0000 & 29,9901 & 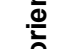 & & PL0-PL9 & 0,0678 \\
\hline & Innorcono & Cone2 & $48,75^{\circ}$ & $48,7442^{\circ}$ & б & & PL0-PL11 & 0,0474 \\
\hline & miner contes & Cone3 & $48,75^{\circ}$ & $48,7316^{\circ}$ & 으 & Perpendicularity & PL45-PL5 & 0,0031 \\
\hline & & & & & F & Coaviality & CYL1 & 0,0062 \\
\hline & & & & & $a$ & Codxidiny & CYL4 & 0,0034 \\
\hline
\end{tabular}

\subsection{Measurement of the gauge with the laser sensor mounted on the CMA}

\subsubsection{Previous studies. Scanning strategy}

Previous studies have played an important role at the time of executing each one of the scanning measurements (tests), as they have aided in the decision of the best scanning strategy to follow in order to obtain the least lack of accuracy possible when comparing the scanned features with the reference ones (CMM). Such studies dealt with the optimal number of scanning "passes" with regard to the lack of accuracy/maximum area covered in the feature [8]. That is, the aim was to reach the maximum accuracy while trying to cover the maximum area of the feature.

To carry out these studies, the example of a sphere was taken as reference. Along different tests, the sphere was scanned using different number of passes (1,2, 3 and 5 passes). Each scanning pass is considered as a single trajectory of the scanner over the target surface in a single direction, avoiding to re-scan the same area already covered by the laser beam since the beginning of the pass. An independent point cloud is generated by each scanning pass. During each pass, the trajectory of the scanner is adapted to the target surface, maintaining an approximate constant distance (close to the scanner stand-off) regarding the surface and orientating the scanner such the laser beam is approximately perpendicular to the surface. Considering the manual nature of this operation, these intentions are impossible to be fulfilled. Furthermore, in a general case, additional passes will be needed to complete the coverage of the whole area of a feature, or to increase the density of the point clouds generated by previous passes.

These tests allow to evaluate the standard deviation of the form error of the sample with regard to the number of points acquired. This number is directly proportional to the number of passes, as the higher the number of passes, the higher the number of points in the sample. In the Fig. 4, the evolution of this parameter is shown. As a result of this survey is has been concluded that the best strategy to perform the scans is only taking 3 passes for each sphere (4 passes for cylinders) covering the major possible area of the feature. 


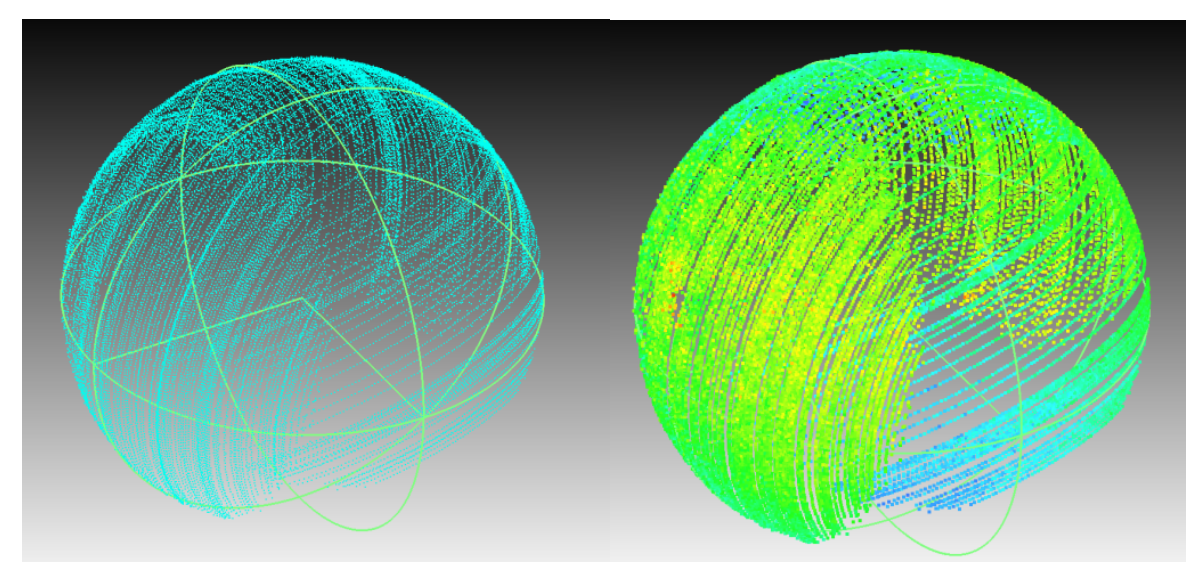

Standard deviation of the sample

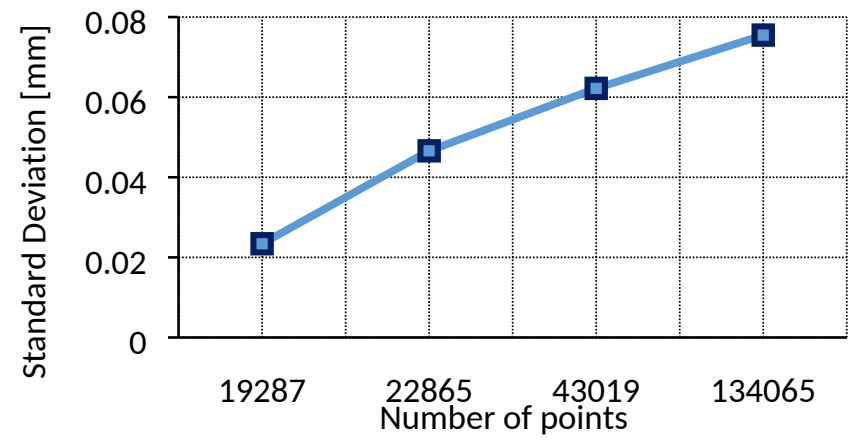

Fig. 4. Measurements evolution obtained from the previous survey tests (without filter).

With relation to the abscise axis on both graphs, the number of points indicated in the graphs correspond in both cases to $1,2,3$, and 5 passes respectively.

Finally, the number of passes selected for scanning spheres was 4 because of the aim of balancing between the percentage of surface area covered in each feature and the lack of accuracy associated to excessive number of passes. In the scanning of planes, only a single pass was necessary in case of ensuring a sufficient coverage of the surface area, whereas for outer cylinders 4 passes were scanned in the direction of the generatrix of the cylinder. In the case of inner cylinders or cones, 5 or 6 passes were required.

Table 4. Best filter and trimming method for each type of feature.

\begin{tabular}{lll}
\hline Type of feature & Previous trimming (min-max number of passes) & Filter \\
\hline Sphere & Below the equator (3-4 passes) & $5 \%$ \\
Planes & For homogenization of areas (1-2 passes) & $5 \%$ \\
Outer cylinders & For homogenization and reduction to 50000 points (4.5 passes) & $10 \%$ \\
Inner cylinders & For homogenization and reduction to 50000 points (4-5 passes) & $5 \%$ \\
Cones & For homogenization and elimination of the cone base (5-6 passes) & $5 \%$ \\
\hline
\end{tabular}

Once the gauge was calibrated with the CMM and the reference values were obtained for each GD\&T dimension, the next step involved measuring the same features but this time by using a laser scanning sensor mounted on the hand of a Coordinate Measuring Arm. All the geometric features involved in the aforementioned dimensions were scanned during 12 measurements (tests), each of which provided a raw point cloud that was subsequently post-processed following a time-consuming procedure.

\subsubsection{Processing of raw point clouds}

For each of the 12 tests, a corresponding raw point cloud comprising all of the gauge features is obtained. In the Fig. $5 a$, a raw point cloud is shown with a great number of spurious points, and points belonging to surfaces of no interest as these surfaces do not pertain to features involved in the dimension being measured. Furthermore, the raw point cloud is referred to an inadequate alignment (observe the location of the reference system trihedron in the lower part of the figure $5 \mathrm{~b}$ ). Therefore, each of the 12 raw point clouds must be treated before the metrological evaluation of the selected dimensions. 
The point cloud treatment, for each one of the 12 tests, was as follows:

- Remove spurious points.

- Raw point cloud trimming (Fig. 5b). The resulting point cloud only contains points belonging to the features of interest for the study.

- Slice the spheres 1 and 6 using a plane located slightly below the equator of the spheres.

- Clean, trim and filter horizontal planes (creating a horizontal medium plane), creating the virtual entities, using the command "Best Shape".

- Alignment (the normal vector of the median plane of prisms top faces defines the $Z$ axis, Sphere 1 and Sphere 6 centres are used to define the $Y$ axis, and the centre of Sphere 1 is established as the origin of the reference system). The coordinate reference system can be seen in Fig. 5b.

- The point clouds were clustered into several sub-point clouds in order to group all the features of the same class (sub-point cloud of outer cylinders, sub-point cloud of inner cylinders, sub-point cloud of cones, etc.). To distinguish these sub-point clouds, they were coloured differently (Fig. 5b and 5c).

- Trimming and filtering of each sub-point cloud. Based on previous studies, different filtering and trimming operations were applied according to the corresponding entity types, as it is collected in Table 4.

- Reconstruction of virtual features. Once the portion of the point cloud corresponding to each feature is filtered, then a "Best Shape" algorithm is applied taking into account the feature type thus generating all the virtual features.

- Measurement of GD\&T parameters applied to the virtual entities (Fig. 5c).

Fig. $5 d$ offers a general view showing the final aspect of the virtual entities after performing the aforementioned operations, where the point clouds have been hidden.

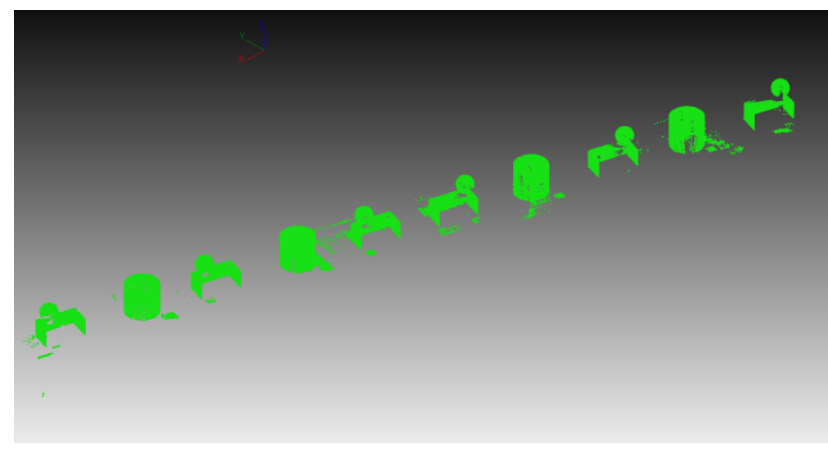

(a)

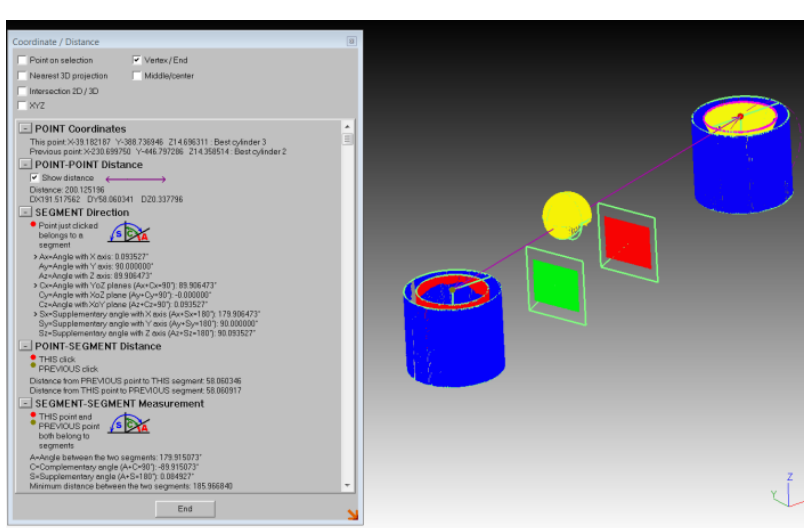

c)

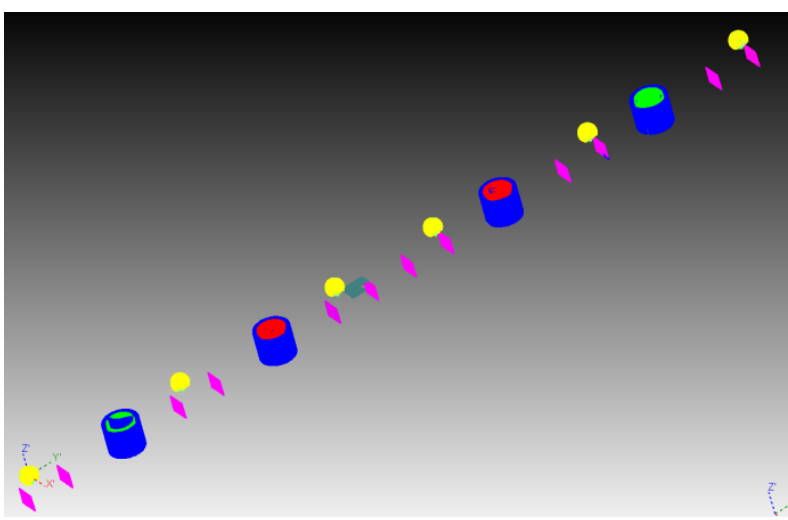

b)

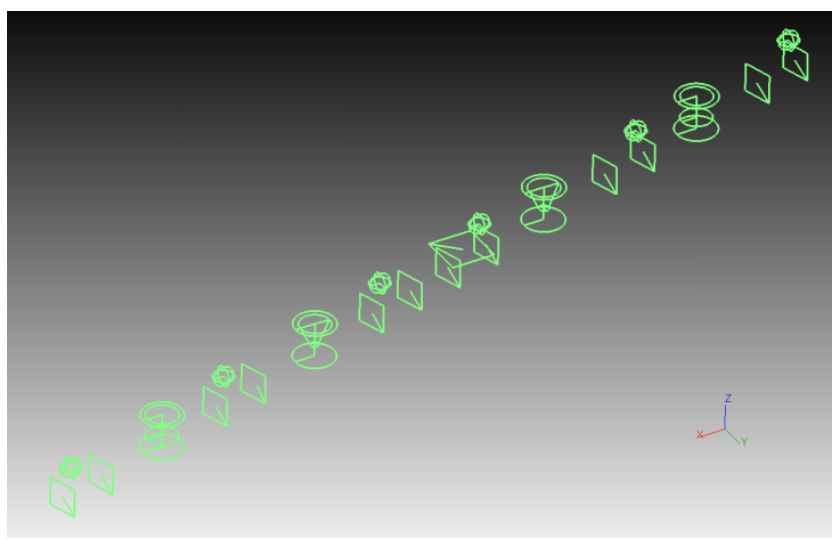

(d)

Fig. 5. Different screenshots of the point cloud processing steps: (a) Raw point cloud. (b) Sub-point clouds after filtering, clustering and alignment. (c) Reconstruction of virtual entities and measurement over virtual entities (3DReshaper®).(d) Generic view of the final virtual entities. 


\subsubsection{Verification of the probing error according to the ISO 10360-12 standard}

During this research, the accuracy of a laser scanning sensor was studied in a similar manner of that proposed by the ISO 10360-12 standard [16], that is, providing a probing error derived from the measurement of a precision sphere. However, the study presented here extended the calibration to the measurement of 6 spheres, measured 12 time each one of them, which supposes a broader study than the one proposed by the ISO standard that only performs one measurement over a single sphere.

The development of the ISO 10360-12 in 2016 supposed the introduction of a brand new regulatory framework for the evaluation of CMAs equipped with a non-contact sensor. Up to that date, the two existing documents for the evaluation of CMAs, the ASME B89.4.22 standard of 2004 and the VDI/VDE 2617-9 technical recommendation of 2009 , only provided procedures for the evaluation of CMAs equipped with contact probes.

The probing error has been defined both for form deviation and for size measurements, and may be determined from reference features such as planes or spheres. In the case of spheres, that is the feature used in this research, the point cloud size must be reduced to 25 points, in a similar way as the one used for determining the probing error of cartesian CMMs equipped with contact sensors according to ISO 10360. Thus, the performance of contact and non-contact sensors may be intercompared.

Following the indications of the standard ISO 10360-8, the upper hemisphere has been divided evenly in 25 regions centred in the 25 points defined in ISO 10360-5 for contact probing systems. For the purpose of this survey, the way to distribute the 25 spherical zones around each one of the 25 points is to increase simultaneously these areas (sphere cap shape) until these areas are tangents to the adjacent areas.

Subsequently the reduction of points may be carried out following any method desired by the user, although the ISO 10360-8 standard suggests three different methods:

1. Select randomly a point lying onto each one of the 25 areas of the sphere.

2. Compute the average coordinates of all of the scanned points within each area.

3. Fit or reconstruct a sphere from the points scanned within each area, and select a point lying onto this sphere.

If the second method is applied, it must be taken into account that the location of the resultant point will be affected by the distribution of the scanned point within each area, whose homogeneity is not highly controlled when the scanning is performed manually, and by the ration between the size of the area and the total area of the sphere. Because of this, the resultant point may lie inside or outside the sphere, which cannot be attached to errors committed by the scanning sensor. Related to this, it can be more useful to evaluate the location of the resultant point in polar coordinates because the average polar radius of all of the points within an area constitutes a good estimation for the polar radius of the resultant point.

On the other hand, if the third method is chosen, problems related with the fitting of spheres from such reduced areas (spherical caps) may arise. It is well known that the error of fitting a point cloud to a sphere is larger when the angle of the covered area is lower. Consequently, this method is not especially robust, apart from being the most complex of the three. This effect is aggravated by the fact of that the greater the angle of incidence between the laser beam and the spherical surface, the lower the number of points captured in that area. Therefore, in the areas close to the equator (with regard to the location of the sensor) the fitting procedure is critical leading to greater errors. 


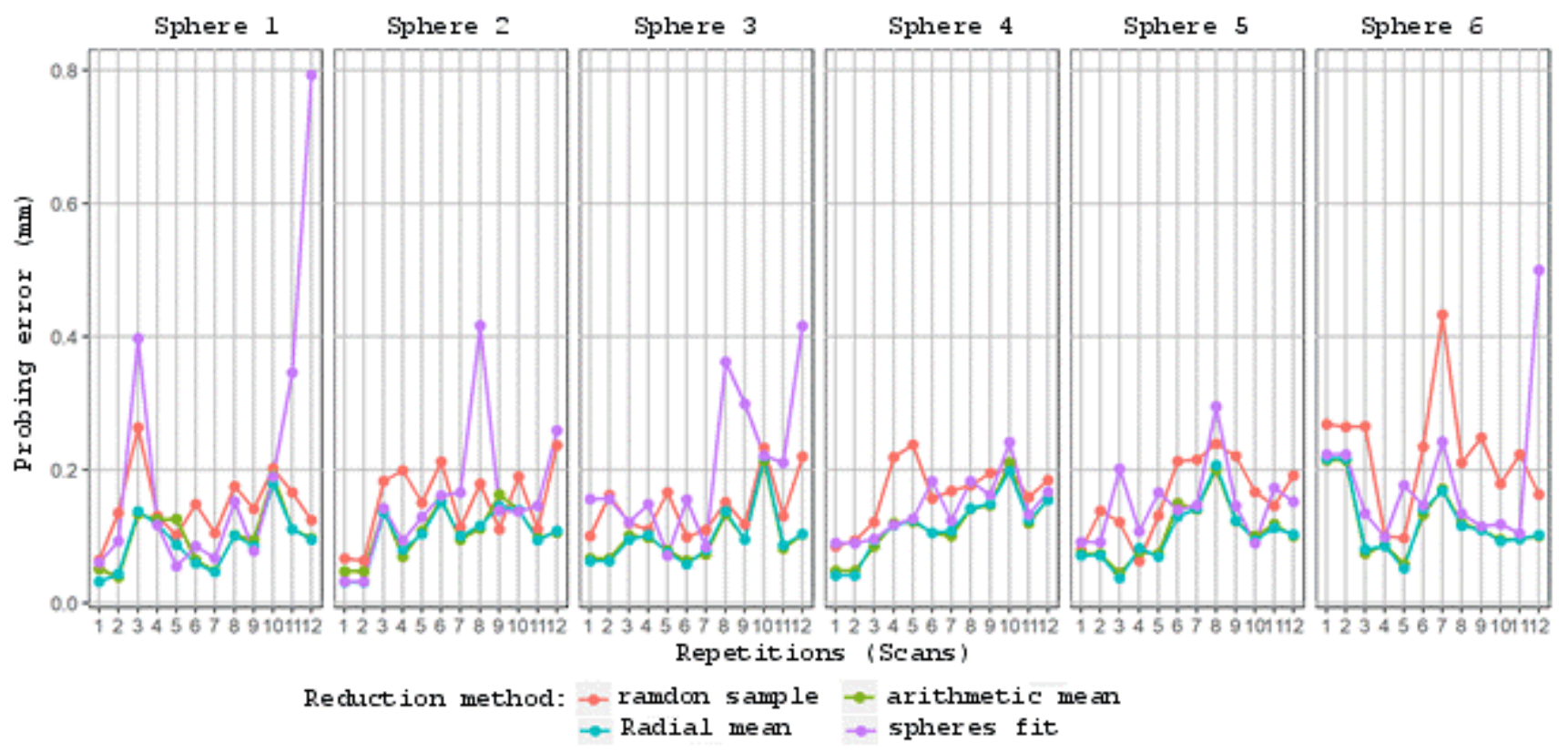

Fig. 6. Several probing errors obtained by different reduction method applied to each of the 12 scans.

Fig. 6 shows the probing error obtained in the 12 measurements of the six spheres. The methods for reducing the point clouds to 25 points correspond to those proposed by the standard, plus the method based on averaging the polar coordinates of the captured point, suggested in this research. Note that the curves corresponding to the "radial mean" and to the "arithmetic mean" reduction methods overlap each other for almost all the represented values.

As it can be deduced from the graphs, the value of the probing error is considerably higher in the case of the random sampling method and in the case of the sphere fitting method, and is lower in the case of the average Cartesian coordinates and in the case of the average polar coordinates methods (Table 5).

Table 5. Mean of probing errors for several reduction methods.

\begin{tabular}{ll}
\hline Method & Probing error $[\mathrm{mm}]$ \\
\hline Random sampling & 0.165 \\
Average cartesian coordinates & 0.109 \\
Average polar coordinates & 0.106 \\
Sphere fitting & 0.173 \\
\hline
\end{tabular}

As conclusion, it can be observed that the methods suggested by the ISO 10360 standard for this type of sensors offer values excessively high and even non-realistic, which is due to the huge effect that a little number of points too separated from the sphere centre has in the calculation of the diameter. This type of equipment with such high "noise" presents high probing errors in the interval of $0.4-0.8 \mathrm{~mm}$. If those spurious points were removed, the probing error would rarely exceed a value of $0.17 \mathrm{~mm}$. In this study, spurious points are not removed in order to appreciate this effect.

\subsubsection{Verification of GD\&T dimensions}

After processing the raw point clouds, a plethora of reconstructed features was obtained. Different types of evaluations were used to analyse the GD\&T dimensions related with each one of these features or with a combination of them (Fig. 5c), evaluations that had been already employed in the measurements carried out with the CMM to establish the reference values for the GD\&T dimensions.

The results of the metrological evaluations will be specified later in another section, where a wide set of graphs and tables will aid in the explanation of all the aspects of the analysis.

Before performing the evaluations, the global set of features was clustered in different groups in order to evaluate the same type of features separately from others. 
The GD\&T dimensions evaluated both from the measurements of the CMM and from the laser sensor were listed and classified in Table 2.

\section{Results}

In this section, the results of the evaluation of GD\&T dimensions are presented. As previously commented in the methodology, the scanned raw point clouds were cleaned, filtered and transformed into virtual features for evaluating GD\&T dimensions of interest. The graphs shown in this section correspond to the metrological evaluation of these GD\&T dimensions related to the virtual features.

Graphs will be classified according to the GD\&T of the Table 2, that is, according to the results for dimensional evaluations, for distance between features, for form deviations and for "combined" tolerances (orientation and location tolerances). Subsequently, the type of each graph will correspond to one of these two models:

- A model that include the results of all of the 12 tests, giving rise a single curve for each GD\&T dimension that is evaluated. For instance, when representing the results about the evaluation of the spheres diameters, the graph will display 6 different curves (1 curve per sphere), each of which will be defined from the 12 measurement results obtained in the tests. A variant of this graph model represents the differences between the measurement results obtained in the 12 tests and the reference value established after measurements with the CMM (Fig. 7 and 8).

- The second model represents a single point for each GD\&T dimension, corresponding to the average value of the measurement results of the 12 tests. For showing the variability between measurement results, an error bar will be also added to the graph at each point. This error bar plots the average plus/minus the standard deviation of the measurement results. In this type of graph, another curve displays the reference values obtained in the measurements with the CMM for comparison purposes. In case of this curve does not appear in the graph, the values represented correspond to differences between measurement results obtained from the laser sensor and from the CMM (Fig. 9).

\subsection{Results of Dimensional analysis}

Figure 7 collects some of the graphs obtained in this research to illustrate the dimensional analysis. Fig. 7 a shows the diameters of the spheres measured with the laser sensor for the 12 tests. For its calculation the Best-Fit algorithm of the 3DReshaper software was used, in this case applying the mentioned filter that removes a $5 \%$ of the points furthest away from the Best-Fit sphere (Table 4). Fig. $7 \mathrm{~b}$ plots the results in the angle measurement of the two available inner cones (approximately $48^{\circ}$ of nominal cone angle). Since the repetitions (samples) are disposed in chronological order, a certain tendency to reduce the repeatability between the first and last scans may even be observed. The experience in the measurement of inner cones was difficult, and even two of the first samples were rejected because of the low coverage of their corresponding point clouds.

Regarding the evaluation of distance measurements, Fig. 8a shows the differences between the results obtained with the laser sensor and the reference values provided by the CMM applied to the measurement of distances between centres of spheres. The values of these differences range between $0.070 \mathrm{~mm}$ and $-0.110 \mathrm{~mm}$. Due to the configuration of the laser integrated in the Coordinate Measuring Arm, as well as the different orientations on each sphere, the distance between the spheres (some are located $200 \mathrm{~mm}$ while others $940 \mathrm{~mm}$ ) seems not relevant for calculating or predicting this deviation. 
Sphere diameters measured by Laser

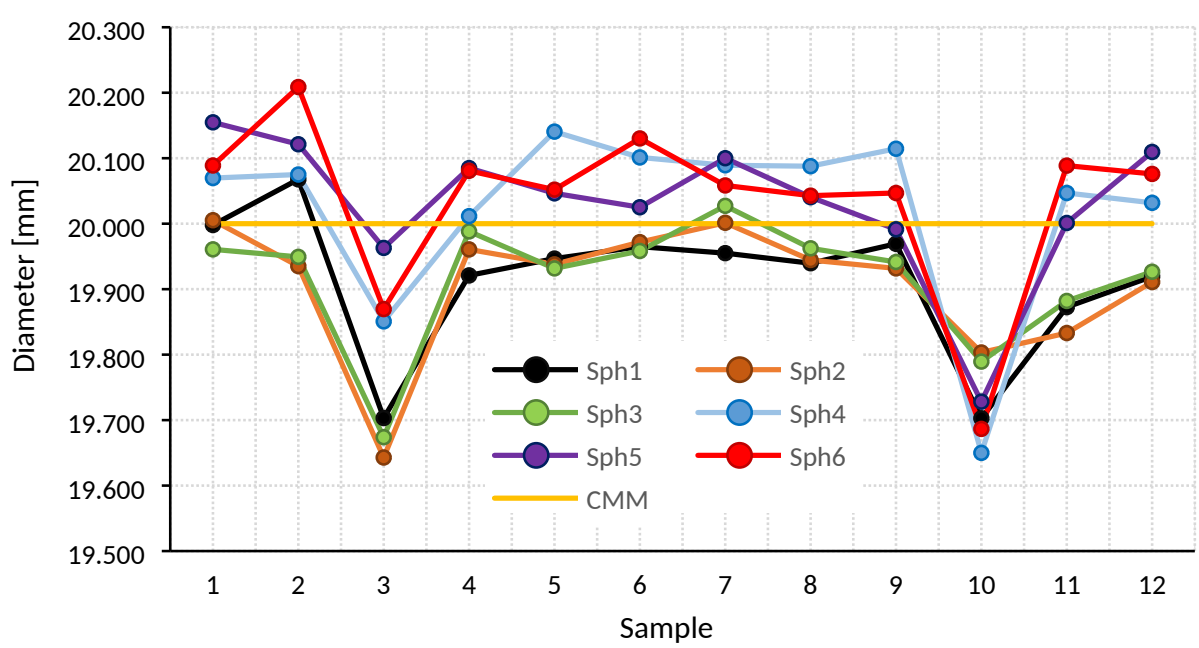

(a)

Angles of cones measured by Laser vs. CMM

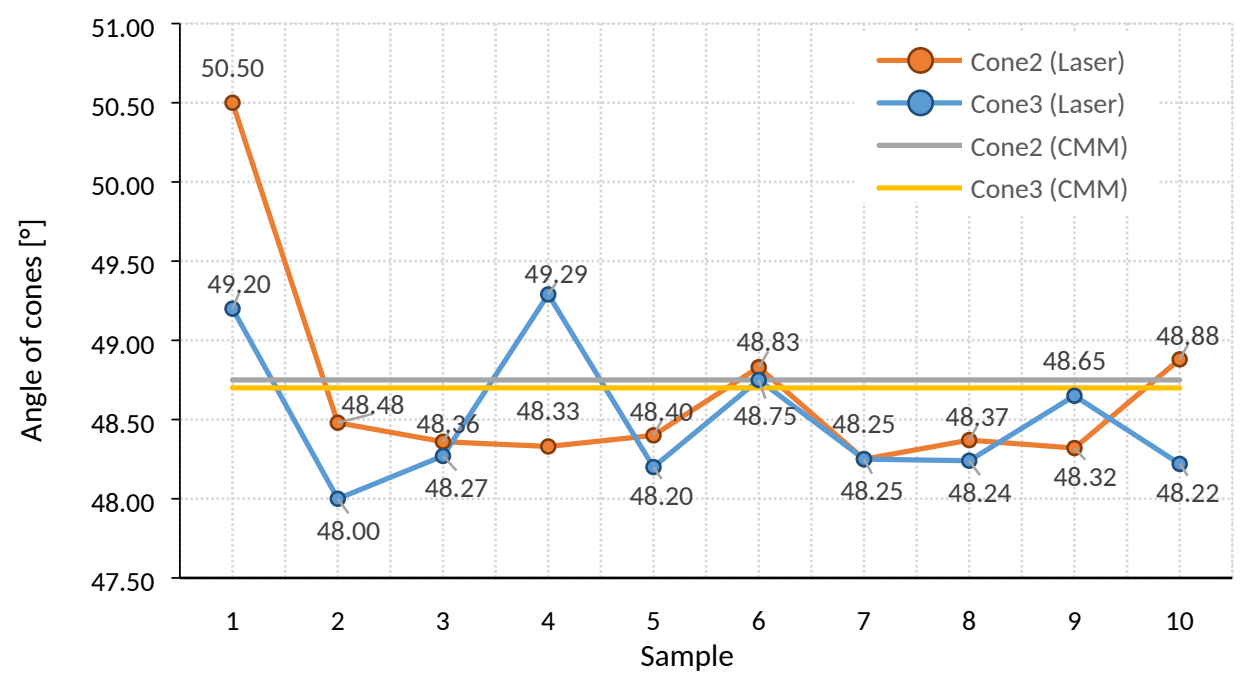

(b)

Fig. 7. Values measured with the laser sensor and the CMM for: (a) Diameter of spheres. (b) Angle of cones.

Fig. $8 \mathrm{~b}$ shows the difference between the distance between planes, measured by the laser and with respect to the $\mathrm{CMM}$. For this calculation, the 2D distance (according to the $\mathrm{Y}$ axis of the alignment reference system) between the centroids of the planes was computed. Similarly, the value of the difference between measurements carried out by the laser sensor and the CM is independent of the value of the distance between planes. However, it can be noted that the value of this difference is always negative, it ranges from $-0.015 \mathrm{~mm}$ in the best of cases to $0.350 \mathrm{~mm}$, in the worst case, meaning that the laser sensor always measures values lower than those provided by the CMM.

In the case of the evaluation of distances between outer cylinder (Fig. 8c), it was taken the value of the distance between the centres of the circles resulting from the intersection of each cylinder with the XY plane of the reference system. The results of the different tests reflect variability between $-0.100 \mathrm{~mm}$ and $+0.094 \mathrm{~mm}$ in the most extreme cases. Again, the differences registered do not depend on the distance at which the cylinders are separated from each other. 
Differences between Laser vs. CMM measurements: Distance between spheres

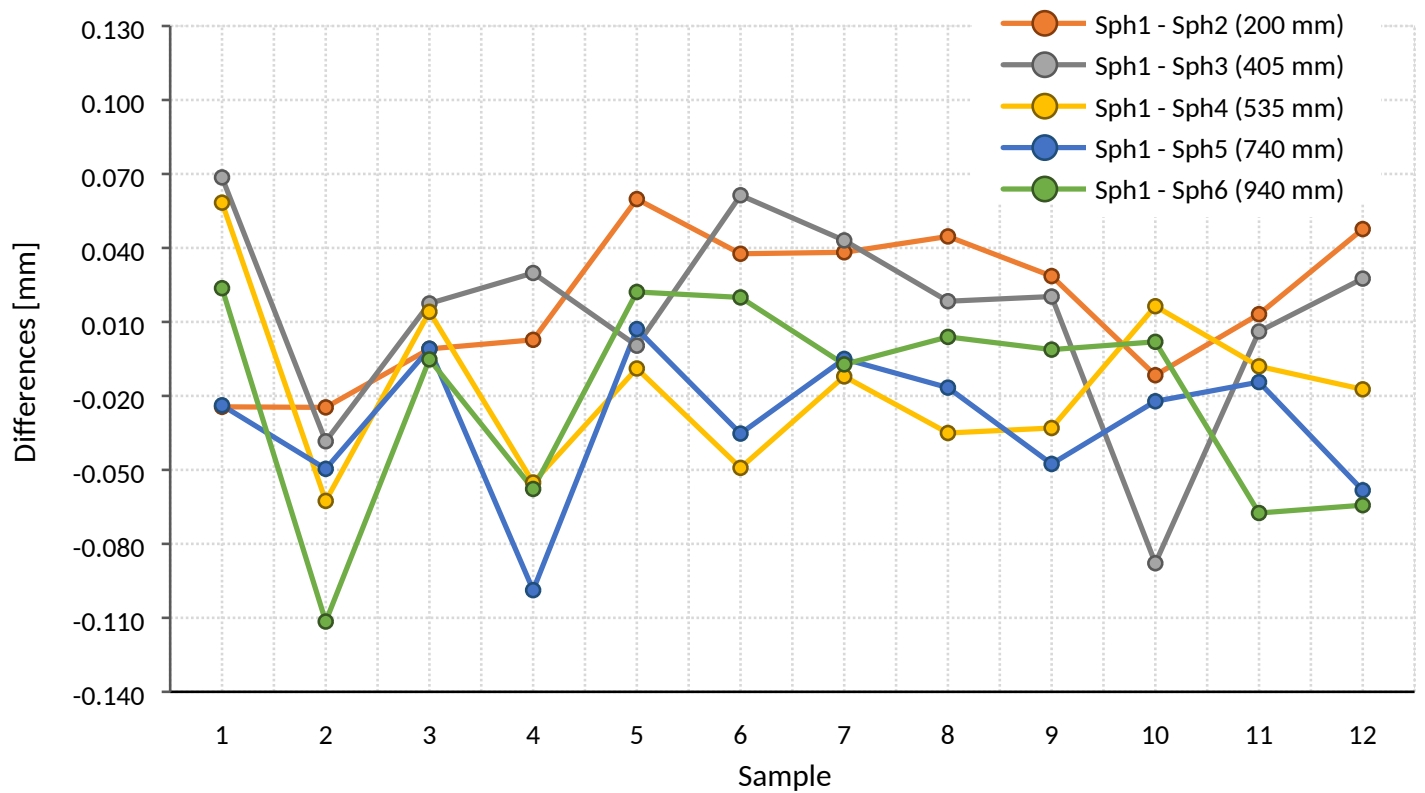

(a)

Differences between Laser vs. CMM measurements: Distance between planes

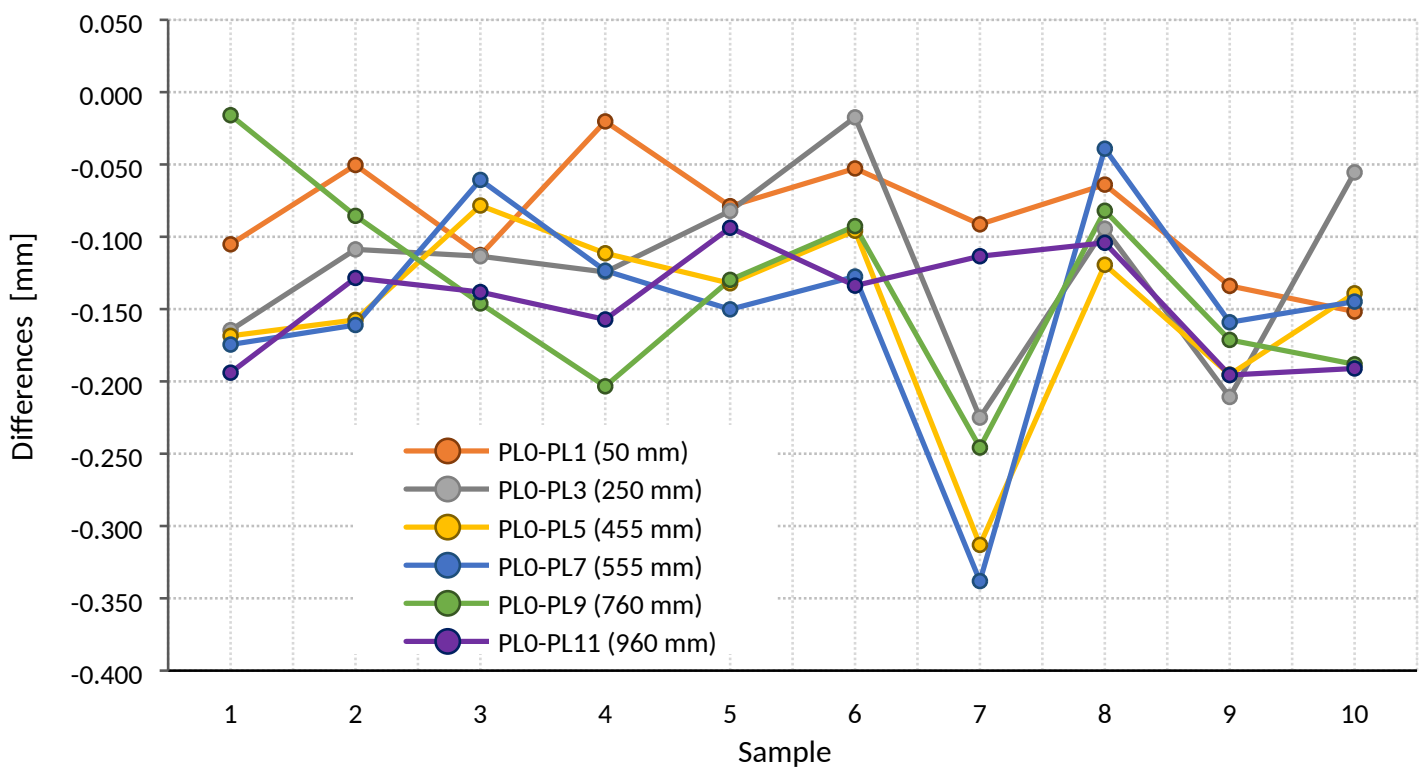

(b) 
Differences Laser vs. CMM measurements: Distance between outer cylinders

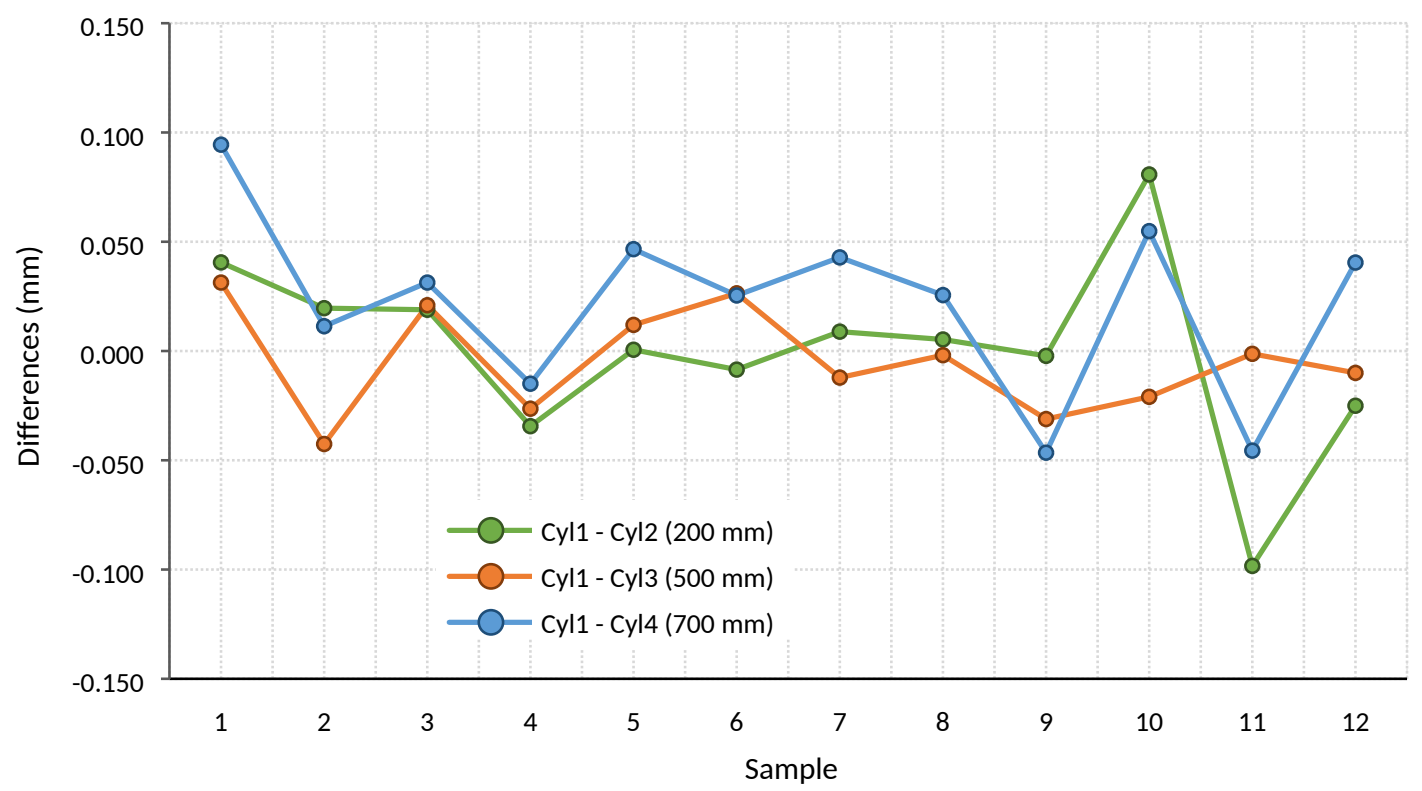

(c)

Fig.8. Differences between measurement results obtained with Laser sensor and CMM for distances between: (a) Centres of spheres, (b) Centroids of planes, and (c) Centres of outer cylinders.

\subsection{Form deviations}

The analysis of form deviations has also been studied in detail. In this case, the graphs are provided according to the second model mentioned above, where both the mean value (as a round point) and the standard deviation (as an error bar at both sides of the mean value) of the 12 measured values obtained for each entity in each of the 12 repetitions. Fig. 9 shows the quality with which the laser sensor is able to capture the different entities, the form deviation of spheres (Fig. 9a), the flatness of planes (Fig. 9b) and the cylindricity of the outer cylinders (Fig. 9c). At the bottom of each graph it was also added the reference value of each form deviation provided by the CMM measurements. Being characteristics of high precision and measured with the CMM, the variability in the average form deviation is one or two orders of magnitude greater than those measured by the CMM.

0

In any case, the average form deviation for spheres varies between 0.030 and $0.050 \mathrm{~mm}$, the flatness of the planes between 0.039 and $0.056 \mathrm{~mm}$ and the cylindricity of the outer cylinders between 0.066 and $0.074 \mathrm{~mm}$. These values are measured with sufficient coverage of points (over 50,000 points/entity), after applying the filters and passes shown in Table 4.

\section{Discussion}

The analysis carried out in this survey has allowed to calculate the metrological limits that Laser sensors mounted on Coordinate Measuring Arms are capable to achieve when they are used for evaluating dimensional and geometric tolerances. Although the R-Scan sensor is a rather obsolete laser scanner model, the methodology proposed in this work is fully valid as it provides a high range of traceable values. 


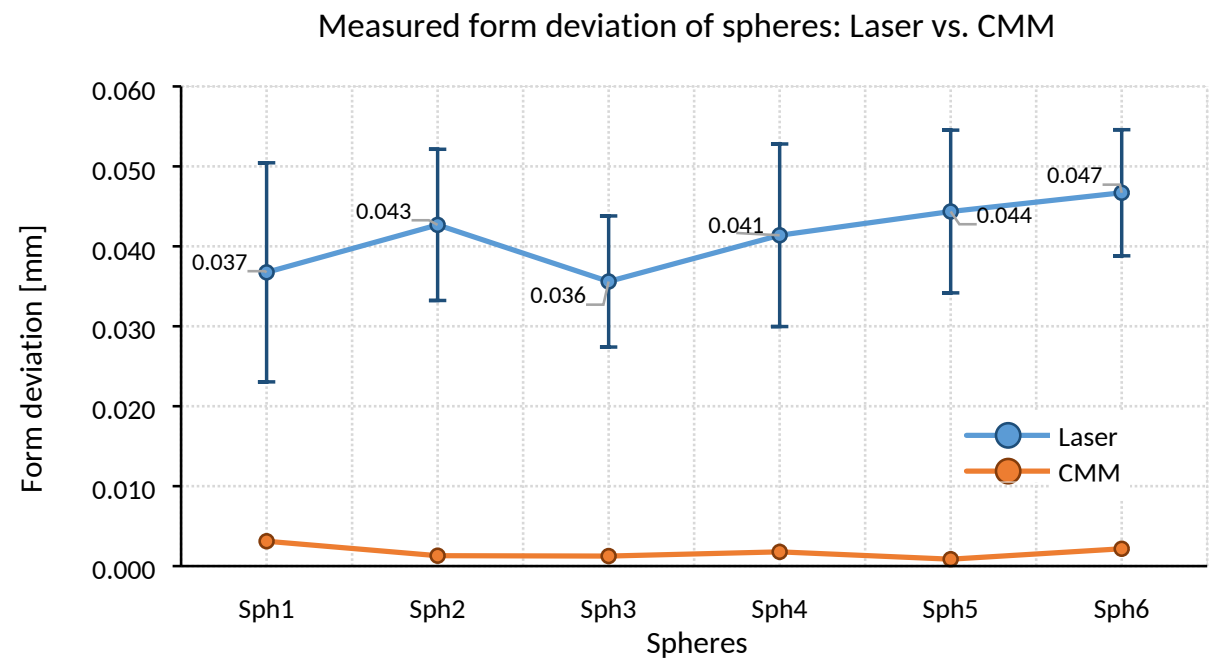

(a)

Measured flatness of planes: Laser vs. CMM

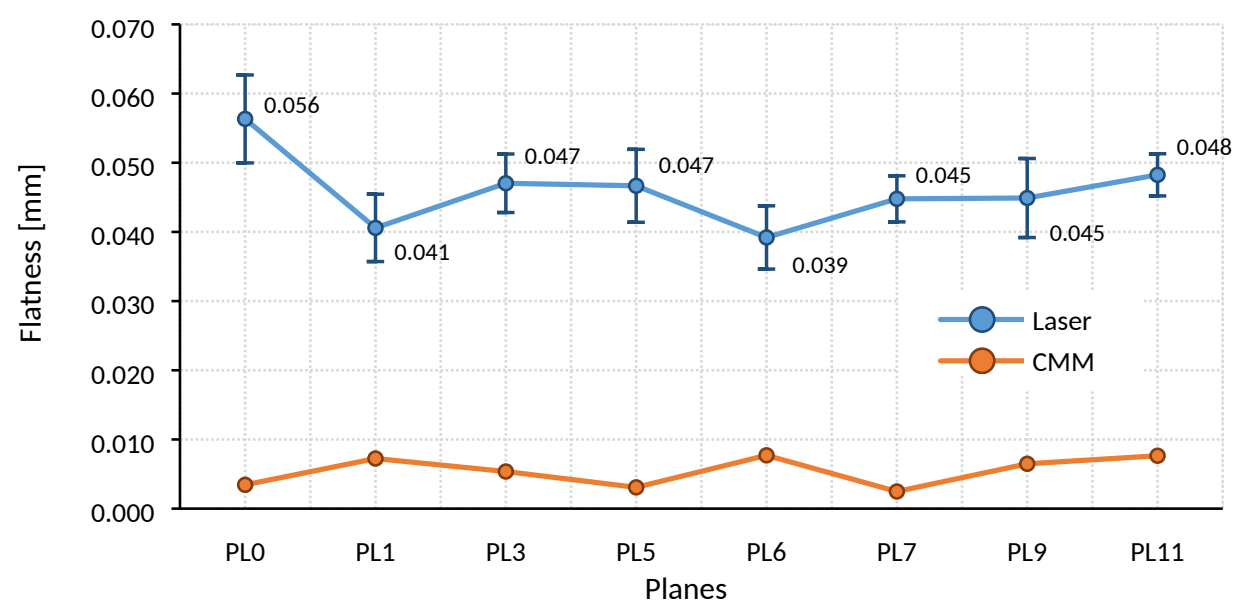

(b)

Measured cylindricity of outer cylinders: Laser vs. CMM

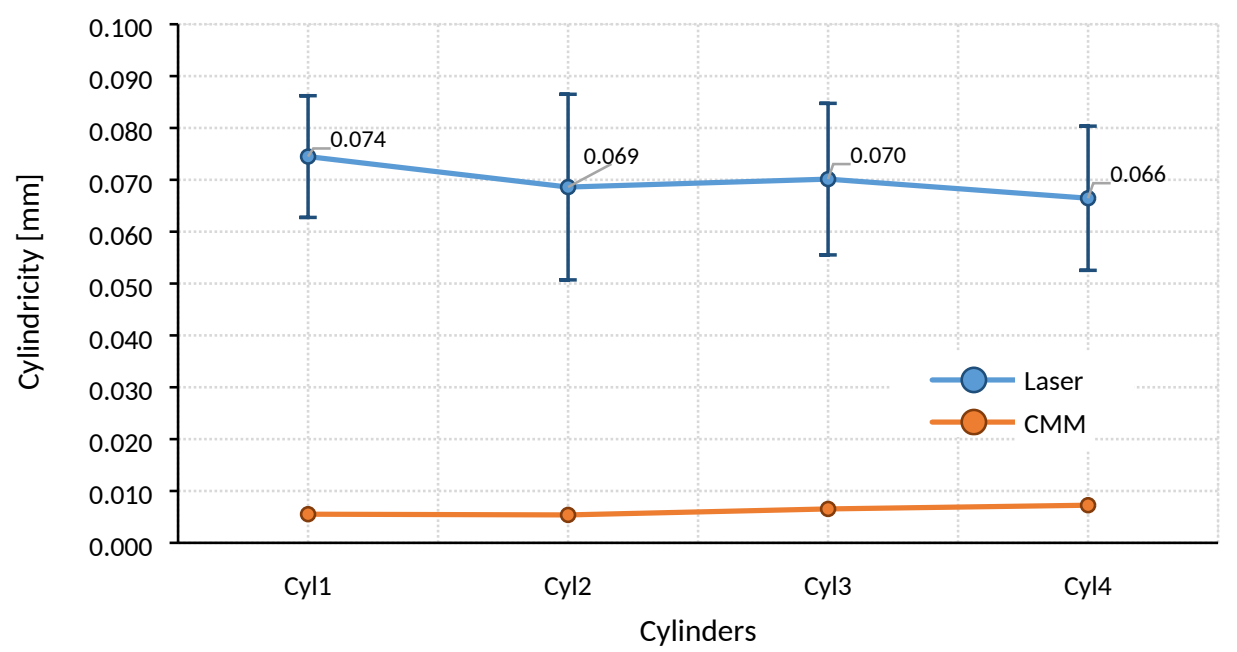

(c)

Fig. 9. Analysis of the form deviation measured with the laser sensor compared with the reference values obtained with the CMM: (a) Spheres form deviation. (b) Planes flatness. (c) Cylindricity of outer cylinders.

One issue that has received special attention is the control of the variability in the measurement results derived from manual scanning operations. In addition, the variability attributable to the completely manual procedure the operator develops when scanning different geometrical entities has been minimized in two ways. On one hand, the scanning strategy has been optimized based on previous studies, by establishing (limiting) the number of 
orientations and scanning passes to access each entity. And, on the other hand, a statistic analysis has been performed from a series of 12 scanning tests, each of one including all the entities of a feature-based gauge. Moreover, as each test comprises the scanning of several items of the same type of entity ( 6 spheres, 4 cylinders, 12 planes, etc.), a precise map of the laser scanner performance for evaluating dimensional and geometric tolerances (GD\&T) is obtained.

Table 6. Means of differences and its standard deviations between Laser and CMM measurements.

\begin{tabular}{|c|c|c|c|}
\hline \multirow[b]{2}{*}{ Evaluation type } & \multirow[b]{2}{*}{ Features involved } & \multicolumn{2}{|c|}{ Laser scanning measurements } \\
\hline & & $\begin{array}{l}\text { Means of Differences } \\
\bar{x}[\mathrm{~mm}]\end{array}$ & $\begin{array}{l}\text { Mean of Standard deviations } \\
\sigma[\mathrm{mm}]\end{array}$ \\
\hline \multirow{4}{*}{$\begin{array}{l}\text { Linear (or angular) } \\
\text { dimensions }\end{array}$} & Spheres diameter & 0.0807 & 0.0681 \\
\hline & Outer cylinders diameter & 0.1166 & 0.0870 \\
\hline & Inner cylinders diameter & 0.0912 & 0.0523 \\
\hline & Inner cones $\left(^{\circ}\right)$ & $0.4509^{\circ}$ & $0.3390^{\circ}$ \\
\hline \multirow{4}{*}{$\begin{array}{l}\text { Distances between } \\
\text { features }\end{array}$} & $1^{\text {st }}$ Sphere (Sph1) to Sphere i-th & 0.0315 & 0.0258 \\
\hline & $1^{\text {st }}$ Plane (PL0) to Plane i-th & 0.1263 & 0.0562 \\
\hline & $1^{\text {st }}$ Outer (Cyl1) to Cyl i-th & 0.0294 & 0.0219 \\
\hline & Inner Cyl1 to Inner Cyl4 & 0.0603 & 0.0390 \\
\hline \multirow[t]{5}{*}{ Form deviations } & Spheres & 0.0412 & 0.0101 \\
\hline & Planes & 0.0405 & 0.0093 \\
\hline & Outer cylinders & 0.0699 & 0.0145 \\
\hline & Inner Cylinders & 0.1116 & 0.0273 \\
\hline & Inner Cones & 0.0654 & 0.0397 \\
\hline \multirow{3}{*}{$\begin{array}{l}\text { Position or orientation } \\
\text { deviation }\end{array}$} & Perpendicularity & 0.0451 & 0.0409 \\
\hline & Parallelism & 0.0287 & 0.0370 \\
\hline & Coaxiality & 0.0389 & 0.0288 \\
\hline
\end{tabular}

Metrological evaluation according GD\&T measurement

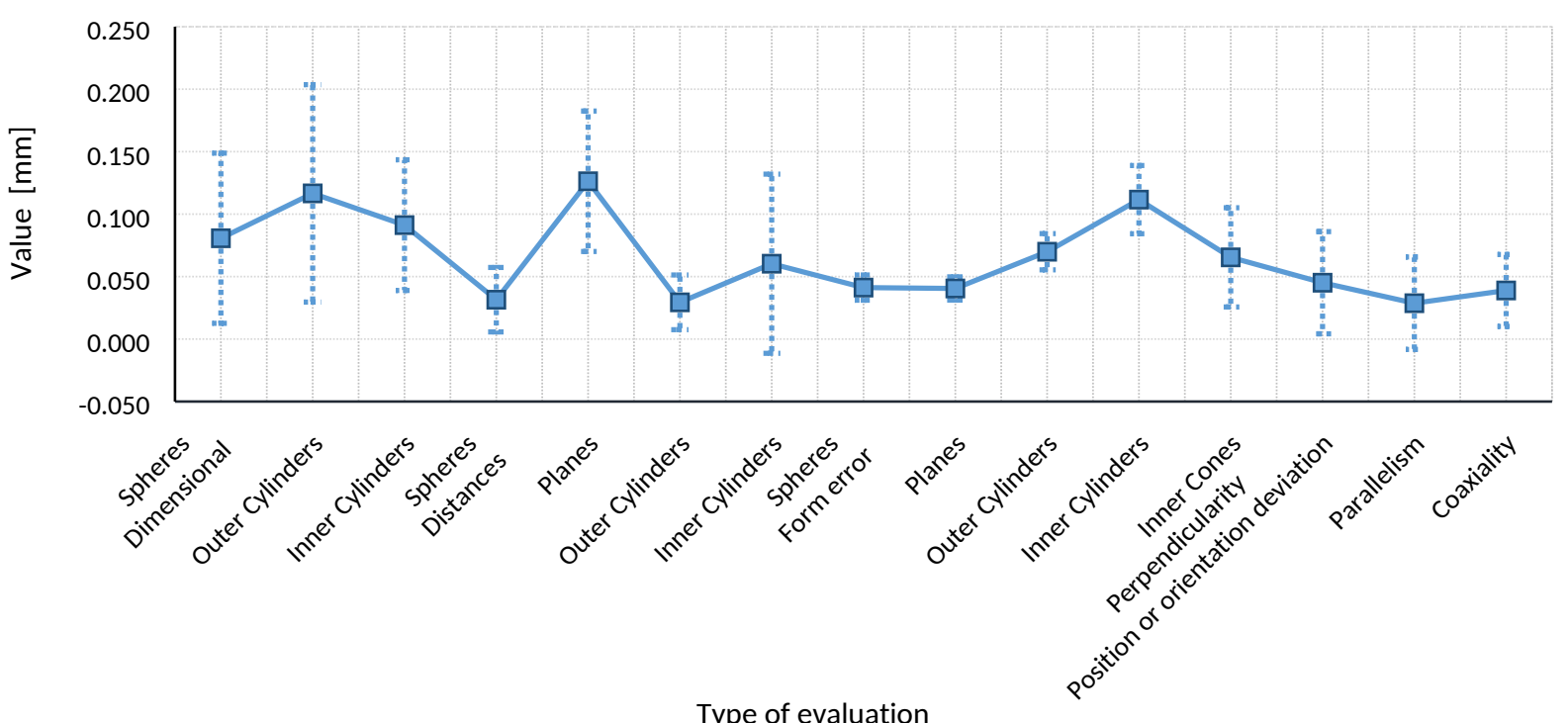

Fig. 10. Global comparison between Laser \& CMM. Aggregation data by type of evaluation.

In view of the work carried out and the type of graphs that have been plotted, the comparison between Laser-CMM can be extended by attempting to carry out a global evaluation (Fig. 10, table 6). This global evaluation incorporates all the previous types of evaluation, allowing to draw conclusions regarding the capacity of the sensor for metrological measurements for GD\&T verification, beyond the reverse engineering applications for which this type of sensor is usually dedicated. 
Thus, the graph shown in fig 10 represents this global evaluation arranging the data according to the GD\&T type, which shows important differences between the values of the mean deviations and the corresponding standard deviations (shown as a vertical error bar) obtained in the different tests. For example, when the scanner is used to measure dimensions, e.g. diameters, mean deviations between 0.11 (outer Cyl) to $0.080 \mathrm{~mm}$ (Sph), and with a high variability (standard deviations between $0.052-0.087 \mathrm{~mm}$ ), are to be expected. If the scanner is otherwise used to evaluate distances, the values improve, especially for distances between spheres, $0,031 \mathrm{~mm}$ (with a low Std. dev. of $0,025 \mathrm{~mm}$ ) or between outer cylinders, $0,029 \mathrm{~mm}$ (std. dev. of $0.021 \mathrm{~mm}$ ), probably because distances are evaluated between the centres of the entities thus compensating the diametrically opposite measures. In the case of distances between planes, the expected differences are higher, in the order of $0.126 \mathrm{~mm}$. In this case, it is probably due to consider, in the same study, both distances between planes far apart (up to $960 \mathrm{~mm}$ ) and between planes located closely $(50 \mathrm{~mm})$, without averaging the position of each entity, as it happens with the spheres. The standard deviation in the measurement of distance between planes is also high $(0.056 \mathrm{~mm})$.

Regarding the form errors of the analysed entities, the value of the deviations is similar in general lines to the dimensional and distances deviations, around $0.040 \mathrm{~mm}$ (planes and spheres) to $0.110 \mathrm{~mm}$ in the worst case (inner cylinders). However, the most remarkable thing is related with the variability in the measurement of form error, as the repeatability reaches values between 0,010 and $0,030 \mathrm{~mm}$ due undoubtedly to the less movement of the AACMM joints (encoders) for capturing individual entities.

As can be seen on the right side of graph (Fig. 10), when the sensor is used to measure location or orientation tolerances, intermediate values $(0.028-0.045 \mathrm{~mm})$ are obtained with deviations of the same order of magnitude. These intermediate values are explained because the associated entities are adjacent, allowing scanning in a relatively small work area (similar to the procedure used for form errors), although the entities measured here force the encoders to rotate to a greater extent than in that case.

\section{Conclusions}

This experimentation has allowed the validation of the optical feature-based gauge developed for this study, proving that it is especially suitable for evaluations more complex and extensive than those proposed by ISO 10360-12, ASME B89.4.22 or VDI 2617-6, whose procedures are largely based on the measurement of distances between only two types of entities (spheres and planes).

Moreover, in this case, a very interesting proposal has been made to evaluate the probing error by scanning precision spheres, without the need to have 100\% coverage (and without the need to have high density) of the upper hemisphere, obtaining a reliable probing error with less coverage, and requiring fewer orientations of the AACMM wrist (laser sensor).

In order to study the variability produced by manual laser scanning operations, during the 12 tests (repetitions) the trajectories of the scanning passes were maintained as similar as a manual process allows, so that between repetitions the variability of the position of the CMA joints was minimised as much as possible, adopting the same relative positions between joints. On the other hand, there are GD\&T measurements that are associated to a greater joint variability than others (from different relative positions between the scanner and the workpiece), as the global graph of Fig. 10 and results on Table 6 reflect.

\section{Acknowledgements}

The authors thank to grants awarded by the University Institute of Industrial Technology of Asturias (IUTA, ref. SV16-GIJON-1-04 and SV-17-GIJON-1-14), and to the Spanish Ministry of Economy and Competitiveness (project DPI2012-36642, through FEDER-ERDF funds).

\section{References}

[1] Feng HY, Liu YX, Xi FF. Analysis of digitizing errors of a laser scanning system. Precision Engineering. 2001; 25:185-191.

[2] Son S, Park HP, Lee KH. Automated laser scanning system for reverse engineering and Inspection. International Journal of Machine Tools \& Manufacture. 2002; 42:889-897. 
[3] Mahmuda M, Joannic D, Roy M, Isheil A, Fontaine JF. 3D part inspection path planning of a laser scanner with control on the Uncertainty. Computer-Aided Design. 2011; 43:345-355.

[4] Van Gestel N, Cuypers S, Bleys P, Kruth JP. A performance evaluation test for laser line scanners on CMMs. Optics and Lasers in Engineering. 2009; 47:336-342.

[5] Zhao H, Kruth JP, Van Gestel N, Boeckmans B, Bleys P. Automated dimensional inspection planning using the combination of laser scanner and tactile probe, Measurement. 2012; 45:1057-1066.

[6] Ding LJ, Dai SG, Mu PA. CAD-Based Path Planning for 3D Laser Scanning of Complex Surface. Procedia Computer Science. 2016; 92:526-535.

[7] Gerbino S, Del Giudice DM, Staiano G, Lanzotti A, Martorelli M. On the influence of scanning factors on the laser scanner-based 3D inspection process, International Journal of Advance Manufacturing Technology. 2016; 84:1887-1799. (doi:10.1007/s00170-015-7830-7).

[8] Besic I, Van Gestel N, Kruth JP, Bleys P, Hodolic J. Accuracy improvement of laser line scanning for feature measurements on CMM. Optics and Lasers in Engineering, 2011; 49:1274-1280.

[9] Santolaria J, Guillomía D, Cajal C, Albajez JA, Aguilar JJ. Modelling and Calibration Technique of Laser Triangulation Sensors for Integration in Robot Arms and Articulated Arm Coordinate Measuring Machines, Sensors 9, 7374-7396; 2009 (doi:10.3390/s90907374).

[10] Rak M, Woźniak A. The influence of properties of a measured object on the surface digitalization performed by a laser scanner integrated with measuring arm, Nauka (Pomiary Automatyka Robotyka). 2012; 12:76-81.

[11] Rak M, Woźniak A, Mayer JRR, The influence of the scanning path of a laser scanner integrated with measuring arm on the surface digitalization, $X^{\text {th }}$ International Scientific Conference Coordinate Measuring Technique, Bielsko-Biała, Ustron, Poland, 23rd - 25th April, 2012. (doi:10.13140/2.1.2115.9045).

[12] Ratajczyk E, Rak M, Kowaluk T. The influence of method of point collection on results with the use of a measuring arm, Metrology and Measurement Systems, Vol. XIX (3), pp. 541-552. 2012.

[13] Korosec M, Duhovnik J, Vukasinovic N. Identification and optimization of key process parameters in noncontact laser scanning for reverse engineering, Computer-Aided Design 42, 744 748, 2010.

[14] Wang Y, Feng HY. Modeling outlier formation in scanning reflective surfaces using a laser stripe scanner, Measurement 57, 108-121. 2014.

[15] Simone C, Enrico S. Metrological performance verification of coordinate measuring systems with optical distance sensors, International Journal of Precision Technology. 2011; 2(2-3):153-171. (doi:10.1504/IJPTECH.2011.039457).

[16] Boeckmans B, Probst G, Zhang M, Dewulf W, Kruth JP. ISO 10360 verification test applied to CMMs equipped with a laser line scanner, Dimensional Optical Metrology and Inspection for Practical Applications, Proc. of SPIE. 2016; 9868:986805-1.

[17] Peternell M. Developable Surface Fitting to Point Clouds, Computer Aided Geometric Design, 2004: $21: 785$ 803.

[18] Cuesta E, González-Madruga D, Alvarez BJ, Barreiro J. A new concept of feature-based gauge for Coordinate Measuring Arm evaluation. Measurement Science and Technology, 2014; 25:06004-13.

[19] González-Madruga D, Barreiro J, Cuesta E, Martínez-Pellitero S. Influence of human factor in the AACMM performance: a new evaluation methodology. International Journal of Precision Engineering and Manufacturing, 2014; 15(7):1283-1291.

[20] Cuesta E, Alvarez BJ, Patiño H, Telenti A, Barreiro J. Testing Coordinate Measuring Arms with a geometric feature-based gauge. In-situ field trials, Measurement Science and Technology, 2016; 27(5):055003-17. (doi:10.1088/0957-0233/27/5/055003).

[21] E. Cuesta et al., Method and feature-based gauge for the calibration and verification of Articulated Arm Coordinate Measuring Machines. Spanish Patent No. ES2490940 B1 (WO2014135721 A1), 2014. 
[22] Martínez-Pellitero S, Cuesta E, Giganto S, Barreiro J. New procedure for qualification of structured light 3D scanners using an optical feature-based gauge. Optics and Lasers in Engineering. 2018; 110:193-206.

[23] MACOR, Ceramic Substrates and Components Ltd., http://www.macor.info/, http://www.ceramicsubstrates.co.uk/machinable-ceramics/macor/ 